\title{
Modeling Academic Performance Evaluation using Fuzzy C-Means Clustering Techniques
}

\author{
Ramjeet Singh Yadav \\ Department of Computer Science and Engineering, \\ (SET), Sharda University, Greater Noida, UP, India.
}

\author{
Vijendra Pratap Singh, \\ Department of Computer Science and Applications, \\ MG Kashi Vidyapith Varanasi, UP, India
}

\begin{abstract}
In this paper we explore the applicability of Fuzzy C-Means clustering technique to student allocation problem that allocates new students to homogenous groups of specified maximum capacity, and analyze effects of such allocations on the academic performance of students. This paper also presents a Fuzzy set and Regression analysis based rules based Fuzzy Expert System model which is capable of dealing with imprecision and missing data that is commonly inherited in the student academic performance evaluation. This model automatically converts crisp sets into fuzzy sets by using CMeans clustering technique for academic performance evaluation.
\end{abstract}

\section{General Terms}

Clustering Technique, Fuzzy Model and Fuzzy C-Means

\section{Keywords}

Fuzzy Logic, Clustering, Fuzzy C-Means Clustering Technique, Rule based Fuzzy Expert Systems, Membership Function and Academic Performance Evaluation.

\section{INTRODUCTION}

The student academic performance evaluation problem can be considered as a clustering problem where clusters (or classes) are formed on the basis of intelligence level of students, and the class size should not exceed the predefined capacity. The intelligence level wise grouping is essential for maintaining the homogeneity of the group otherwise it would be difficult to provide good educational services to highly diverse student population. Moreover, homogenous grouping of students having similar ranking (or some other measures) into classes would further make the academic performance results fairer, realistic and comparable.

The existing practice of score aggregation based student similarity or his/her rank determination is unrealistic because scores are assembled from different score combinations. Universities use GPA (Grade Point Average), an example of score aggregation based measure, as a major criterion for student selection. Most universities consider 3.0 and above GPA as an indicator of good academic performance, hence, it remains the most common factor used by the academic planners to evaluate progression in an academic environment [23] — despite its limitations in providing a comprehensive view of the state of students' performance evaluation and simultaneously discovering important details from their continuous performance assessments [24]. Furthermore, average score may lead to wrong conclusion. Especially, when details of data from which it is computed are not given.

It has been observed that there are factors, other than academic ones, pose barriers to students attaining and maintaining high. Therefore, grouping or clustering students using cognitive as well as affective factors into different categories, and then defining performance measure may be a realistic approach. For example, consider a scenario where two students score 50, 60, 70 , and 70,60, 50 in three tests respectively. The average mark obtained by each is 60 . Can we conclude, from the average, that intelligence level of both the students is same? Of course not! The data indicates that one student is improving while the other is deteriorating consistently-it may imply that one student is learning consistently from his experience.

The example illustrates that the student ranking or modeling academic performance evaluation method should be based on class homogeneity- a view point supported by other researchers [25]. In addition to such computational issues, as mentioned before, the imprecision and vagueness in data collection process also affect the performance indicators evaluation. Unfortunately, this aspect is ignored in practice because generally hard computing based process, procedures and techniques are used in performance evaluation. Observation shows the soft computing techniques are more powerful and better suited in providing feasible solutions to the problems that deal with uncertainties and vagueness. For instance, the fuzzy logic, handles, imprecision, and uncertainty in a natural manner by providing a human oriented knowledge representation is possible, but it is weak in self learning and generalization of rules. A combination of fuzzy logic and genetic algorithm is expected to eliminate this weakness. Now, their power is being investigated.

In their recent work, Mankad, Sajja and Akerkar have reported an evolving rule based model for identification of multiple intelligence [1]. Their genetic-fuzzy hybrid model identifies human intelligence. Zainudin Zukhri and Khairuddin Omar have reported successful application of Genetic Algorithm for solving difficult optimization problems in new students' allocation problem [25]. Vuda Sreenivasarao has developed a model for improving academic performance evaluation of students based on data warehousing and data mining techniques that use soft-computing intensively [27]. Their analysis indicates that the group homogeneity improves students academic performance thereby enhances education quality.

An Artificial Neural Network (ANN) model reported in Afoayan and Shamir Absalom that along with computation also derives meaning from imprecise data, extracts patterns and detects trends [28]. This ability has added new dimensions in comprehending the complex phenomena that is buried in students' data otherwise might have gone unnoticed using hard computing techniques.

In practice, whether phenomena discovery or performance indicator computation, their accuracy depends on the data quality that in turn depends on the accuracy of data collection process and representation techniques. In order to address the data related issues, in education domain, Biswas suggested use of fuzzy sets (Zadeh) in students' answer-sheets evaluation [2, 3]. Wang H.Y. and Chen S.M. recommended that the use of vague sets (Gau and Buehrer) instead of fuzzy sets to represents the vague marks of each question where the evaluator can use vague values to indicate the degree of the evaluators' satisfaction for each question $[4,5]$.

In fuzzy sets the membership evaluation (characteristics function definition) is a major issue. In order to apply the fuzzy 
set in education domain effectively, there have been a lot efforts in defining the effective membership. Bai S.M. and Chen S.M. have defined fuzzy membership functions for fuzzy rules [6]; Law C.K. has used fuzzy numbers [7], and for more information on this issue consult: Chen and Lee [8], Chiu-Keung Law [7], Wang and Chen [9], Stathacopoulou [10], Guh [11], Gokmen [12], Hameed 13], Sirigiri Pavani [26], Neogi [30], Yadav [31], Gupta [18], Krzysztof [20], Mamatha [33], Chaudhari [29], Daud [32], Baylari and Montazer [14], Posey and Hawkes [15], Stathacopoulou [16], Bhatt and Bhatt [17], and Zhou and Ma [19]. The research works cited in the preceding paragraph indicates that the fuzzy logic, neural network and fuzzy neural network have already been employed in student modeling systems but almost nothing or very little has been mentioned about automatic generation of fuzzy membership function. This paper describes a method for automatic generation of membership function for student academic performance evaluation. For this purpose we have used fuzzy C-means Clustering algorithm for automatic generation of membership function. In order to obtain the homogeneous clusters (or classes) of students, we have studied the performance of Fuzzy C-Means and K-Means clustering algorithms for student population clustering. For both the cases, we have developed students' academic performance evaluation models.

In this research paper, the proposed Rule Based Fuzzy Expert System automatically converts the crisp data into fuzzy set and also calculates the total mark of a student appeared in semsete-1 and semester-2 examination. The proposed idea is a starting attempt to use the applicability of Fuzzy C-Means clustering algorithm to analyze and find out modeling academic performance and to improve the quality of the students and teachers performance in educational domains. Fuzzy C-Means Clustering algorithm is a data warehousing and data mining techniques. Due to this reason it is more effective for improve the quality of education. The management can use some techniques to improve the course outcome according to the improve knowledge. Such knowledge can be used to give a good understanding of student's enrollment pattern in the course under study, the faculty and managerial decision maker in order to utilize the necessary steps needed to provide extra classes. On the other hand, such types of knowledge the management system can be enhance their policies, improve their strategies and improve the quality of the system.

The paper, besides introduction, has eight sections. The next section gives a Fuzzy Logic System Works. Section three describes the Data Cluster Analysis for Academic Performance Evaluation. Section four describes the Fuzzy C-Means Clustering Technique. Section five describes the Regression Model and their functioning. Section six describes the architecture of proposed rule based Fuzzy Expert System. Section seven describes the experimental result of proposed rule based Fuzzy Expert System. We conclude paper with section eight.

\section{FUZZY LOGIC SYSTEM WORKS}

Fuzzy logic was invented by Zadeh for handling uncertain and imprecise knowledge in a real world application [3]. It refers to a mode of reasoning in the presence of imprecise or ambiguous information. Fuzzy logic is close to human thinking or reasoning as a natural language. Fuzzy logic provides a simple way to arrive at a definite conclusion based upon vague, ambiguous, imprecise, noisy, or missing input information. It consists of four cardinal components: Fuzzification, a knowledge base, rule base, a decision making mechanism and defuzzification. Figure 1 shows the Fuzzy logic system and their functioning.

\subsection{Membership Function}

The membership function is a graphical representation of the magnitude of participation of each input [37]. A graph that defines how each point in the input space is mapped to membership value between 0 and 1 . Input space is often referred as the universe of discourse or universal set, which contain all the possible elements of concern in each particular. It associates a weighting with each of the inputs that are processed, define functional overlap between inputs, and ultimately determines an output response. The rules use the input membership values as weighting factors to determine their influence on the fuzzy output sets of the final output conclusion. Once the functions are inferred, scaled, and combined, they are defuzzified into a crisp output which drives the system.

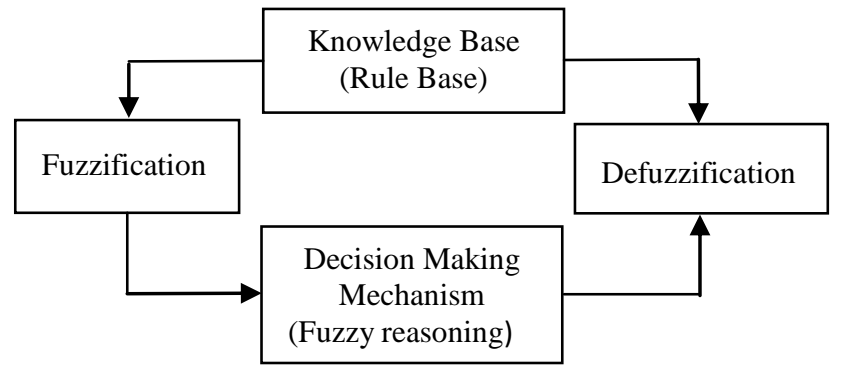

\subsection{Fuzzification}

Figure 1: Fuzzy logic

The Fuzzification comprises the process of transforming crisp values into grades of membership for linguistic terms of fuzzy sets. The membership function is used to associate a grade to each linguistic term [37]. It also refers to as the transformation of an objective term into a fuzzy concept.

\subsection{Fuzzy Rule Base (Knowledge Base)}

Fuzzy logic classification systems are generally implemented in the form of an expert decision support system. The rule base contains the rules and forms the major part of the complete knowledge embedded in the system. Mostly the rules are supplied by the domain expert.

\subsection{Fuzzy Rule Evaluation (Inferencing)}

This step is to determine the firing strength of each rule. The logical products for each rule must be combined or inferred before being passed on to the defuzzification process for crisp output generation. Several inference methods exist; the maxmin method tests the magnitudes of each rule and selects the highest one. The horizontal coordinate of the "fuzzy centroid" of the area under that function is taken as the output. This method does not combine the effects of all applicable rules but does produce a continuous output function and is easy to implement. The max-dot or max-product method scales each member function to fit under its respective peak value and takes the horizontal coordinate of the "fuzzy" centroid of the composite area under the function(s) as the output. This method combines the influence of all active rules and produces a smooth, continuous output. Others are the averaging method and the root-sum-square (RSS) method.

\subsection{Defuzzification}

When the inferencing is over. There is need to compute a single value to represent the outcome. This process is called defuzzification. This can be achieved by different methods. A common method is the defuzzification of the data into a crisp output accomplished by combining the results of the inference process and then computing the "fuzzy centroid" of the area The weighted strengths of each output member function is multiplied by their respective output membership function 
center points and summed. Finally, this area is divided by the sum of the weighted member function strengths and the result is taken as the crisp output.

\section{DATA CLUSTER ANALYSIS FOR ACADEMIC PERFORMANCE \\ EVALUATION}

The clustering problem can be stated simply as follows: Given a finite set of data, $X$, develop a grouping scheme for grouping the objects into classes. In classical cluster analysis, these classes are required to form a partition of $X$ such that the degree of association is strong for data within blocks of the partition and weak for data in different blocks. However, this requirement is too strong in many practical applications, and it is thus desirable to replace it with a weaker requirement. When the requirement of a crisp partition of $X$ is replaced with a weaker requirement of a fuzzy partition or a fuzzy pseudo partition on $X$, we refer to the emerging problem area as fuzzy clustering. Fuzzy pseudo partitions are often called fuzzy c-partitions, where c designates the number of fuzzy classes in the partition [21].

Pattern recognition techniques can be classified into two broad categories: unsupervised techniques and supervise techniques. An unsupervised technique does not use a given set of unclassified data, whereas a supervised technique uses a dataset with known classification. These two types of techniques are complementary to each other. The Hard C-Means and Fuzzy CMeans clustering techniques fall in unsupervised category. In this paper, we have used Fuzzy C-Means clustering techniques for students' academic performance evaluation.

\section{FUZZY C-MEANS (FCM) CLUSTERING TECHNIQUE}

The fuzzy C-Means algorithm (FCM) generalizes the hard CMeans algorithm to allow a point to partially belong to multiple clusters. Therefore, it produces a soft partition for a given dataset. In fact, it produces a constrained soft partition [22]. To this, the objective function $J_{l}$ of hard C-Means has been extended in two ways:

1. The fuzzy membership degrees in clusters were incorporated into the formula.

2. An additional parameter $\mathrm{m}$ was introduced as a weight exponent in the fuzzy membership.

The extended objective function, denoted $\mathrm{J}_{\mathrm{m}}$, is

$J_{m}(P, V)=\sum_{i=1}^{k} \sum_{x_{k} \epsilon X}\left(\mu_{C_{i}}\left(x_{k}\right)\right)^{m}\left\|x_{k}-v_{i}\right\|^{2}$

Where $P$ is a fuzzy partition of the dataset $X$ formed by $C_{1}, C_{2}, \ldots \ldots \ldots, C_{k}$. The parameter $m$ is a weight that determines the degree to which partial members of a cluster affect the clustering result. Like hard c-means, fuzzy c-means also tries to find a good partition by searching for prototypes $v_{i}$ that minimize the objective function $J_{m}$. Unlike hard C-means, however, the fuzzy C-means algorithms also need to search for membership functions $\mu_{C_{i}}$ that minimize $J_{m}$. To accomplish these two objectives, necessary conditions for local minimum of $J_{m}$ was derived from $J_{m}$ are given below in theorem 4.1.

The fuzzy C-means (FCM) algorithm is given below:

$\mathrm{FCM}(\mathrm{X}, \mathrm{c}, \mathrm{m}, \varepsilon)$

$X:$ An unlabeled data set

$\mathrm{C}$ : the number of clusters to form

$\mathrm{m}:$ the parameter in the objective function

$\varepsilon:$ A threshold for the convergence criteria

Initialize prototype $V=\left\{v_{1}, v_{2}, \ldots \ldots \ldots, v_{c}\right\}$

Repeat

$V^{\text {Previous }} \leftarrow V$

Compute membership function using equation (2).

Update the prototype, $v_{i}$ in $\mathrm{V}$ using equation (3).

Until $\sum_{i=1}^{C}\left\|v_{i}^{\text {previous }}-v_{i}\right\| \leq \varepsilon$
Until convergence criteria is met.

\subsection{Fuzzy C-Means Theorem}

A constrained fuzzy partition $\left\{C_{1}, C_{2}, \ldots \ldots \ldots \ldots \ldots, C_{k}\right\}$ can be a local minimum of the objective function $\mathrm{Jm}$ only if the following conditions are satisfied:

$$
\begin{aligned}
& \mu_{C_{i}}(x)=\frac{1}{\sum_{j=1}^{k}\left(\frac{\left\|x-v_{i}\right\|^{2}}{\left\|x-v_{j}\right\|^{2}}\right)^{\frac{1}{m-1}}} \quad 1 \leq i \leq k, x \in X \\
& v_{i}=\frac{\sum_{x \in X}\left(\mu_{C_{i}}(x)\right)^{m} \times x}{\sum_{x \in X}^{n}\left(\mu_{C_{i}}(x)\right)^{m}} \quad 1 \leq i \leq k
\end{aligned}
$$

Bases on this theorem, FCM updates the prototypes and the membership function iteratively using equation (2) and (3) until a convergence criterion is reached. We describe the algorithm in section 4 .

\section{REGRESSION MODEL}

Regression is one of the most common problems in statistics. It consists in exploring the association between dependent and independent variables and in identifying their impact on the dependent variable. Ordinarily, we do not have knowledge of the exact functional relationship between the two random variables $\mathrm{x}$ and $\mathrm{y}$, where to each vector $\mathrm{x}$ sampled according to a distribution $\mathrm{P}(\mathrm{x})$ there corresponds a scalar in accordance to a conditional distribution $\mathrm{P}(\mathrm{y} / \mathrm{x})$. Typically we proceed by assuming that the target variables $\mathrm{y}$ is given by some deterministic function of $\mathrm{x}$ with added Gaussian noise $\epsilon$ that represents a measurement error or, more generally, our "ignorance" about the dependence of $y$ on $x$ ( $H$. White, 1989)[34]:

$y=f_{0}(x)+\epsilon$

The function $f_{0}($.$) is called the regression function and the$ statistical model described by the above equation is called regression model. The error $\epsilon$ is a random variable having a normal distribution with zero mean, and a standard deviation $\sigma$ which does not depend on $\mathrm{x}$ or $\mathrm{y}$, that is:

$$
P(\epsilon)=\frac{1}{2 \pi \sigma^{2}} \exp \left[-\frac{\epsilon^{2}}{2 \sigma^{2}}\right]=\frac{1}{2 \sigma^{2}} \exp \left[-\frac{\left(y-f_{0}(x)\right)^{2}}{2 \sigma^{2}}\right]
$$

This common assumption can be partly justified by results from experimental measurements and by the central limit theorem, which states that the sample mean of any reasonable distribution can be approximated by a normal distribution. It follows from this assumption and from (4) that the conditional distribution of $\mathrm{y}$ given $\mathrm{x}$ will be a normal distribution with mean $f_{0}(x)$ and variance $\sigma^{2}$. Hence we obtain:

$f_{0}(x)=E[y / x]=\int y d x P(y / x)$

That is $f_{0}(x)$ is the conditional mean of the output $y$ given the input $\mathrm{x}$. In other words, the regression of $\mathrm{y}$ on $\mathrm{x}$ is that (deterministic) function of $\mathrm{x}$ that gives the mean value of $\mathrm{y}$ conditional on $x$. It can be demonstrated that the regression function is an excellent solution to the problem of fitting the data, i.e. among all functions of $x$, the regression is the best predictor of $y$ given $x$, in the squared-error sense. Precisely, it can be shown that the minimum of the risk functional:

$R(w)=\int(y-f(x, w))^{2} d P(x, y)$

Is attained by the regression function $f_{0}($.$) . Thus the problem$ of regression estimation can be addressed in the statistical learning framework, once the learning machine is assessed by a quadratic loss function:

$L(y, f(x, w))=(y-f(x, w))^{2}$

In the case of a quadratic loss function, the empirical risk functional becomes:

$R_{E M P}(w)=\frac{1}{N} \sum_{t=1}^{N}(y(t)-f(x(t), w))^{2}$ 
Which is usually referred to as the Mean Squared Error (MSE)? This regression model is used to estimate the output of proposed rule based Fuzzy Expert System.

\section{ARCHITECTURE OF PROPOSED RULE BASED FUZZY EXPERT SYSTEM}

In this paper, we have proposed rule based Fuzzy Expert System for student academic performance evaluation. The proposed rule based Fuzzy Expert System consists of Fuzzy Logic, Fuzzy Cmeans clustering algorithm and Regression analysis model. The Fuzzy C-Means clustering algorithm is used for classify input space into different classes or clusters and regression analysis model used for output estimation of the input data.

\subsection{Rule Based Fuzzy Expert System}

The world of information is surrounded by uncertainty and imprecision. The human reasoning process can handle inexact, uncertain, and vague concepts in an appropriate manner. Usually, the human thinking, reasoning, and perception process cannot be expressed precisely. These types of experiences can rarely express or measured using statistical or probability theory. Fuzzy logic provides a framework to model uncertainty, the human way of thinking, reasoning, and the perception process. Fuzzy system was introduced by Zadeh [3]. A fuzzy expert system is simply an expert system that uses a collection of fuzzy membership functions and rules, instead of Boolean logic, to reason about data [36]. The rules in a fuzzy expert system are usually of a form similar to the following: If $A$ is Low and $B$ is High then $(X=$ Medium $)$. Where $A$ and $B$ are input variables, $X$ is an output variable. Here low, high and medium are fuzzy sets defined on $A, B$ and $X$ respectively. The antecedent (the rule's premise) describes to what degree the rule applies, while the rule's consequent assigns a membership function to each of one or more output variables.

Let $X$ is a space of objects and $x$ be a generic element of $X$. A classical set $A, A \subseteq X$, is defined as a collection of elements objects, such that $\mathrm{x}$ can either belong or not belong to the set. A Fuzzy set $A$ in $X$ is defined as a set of ordered pairs: $A=$ $\left\{\left(x, \mu_{A}(x)\right), x \in X\right\}$, where $\mu_{A}(x)$ is called the membership function (MF) for the fuzzy set A. The MF maps each element of $X$ to a membership grade (or membership value) between zero and one. Figure-2 shows the basic architecture of proposed rule based Fuzzy Expert System for academic performance evaluation.

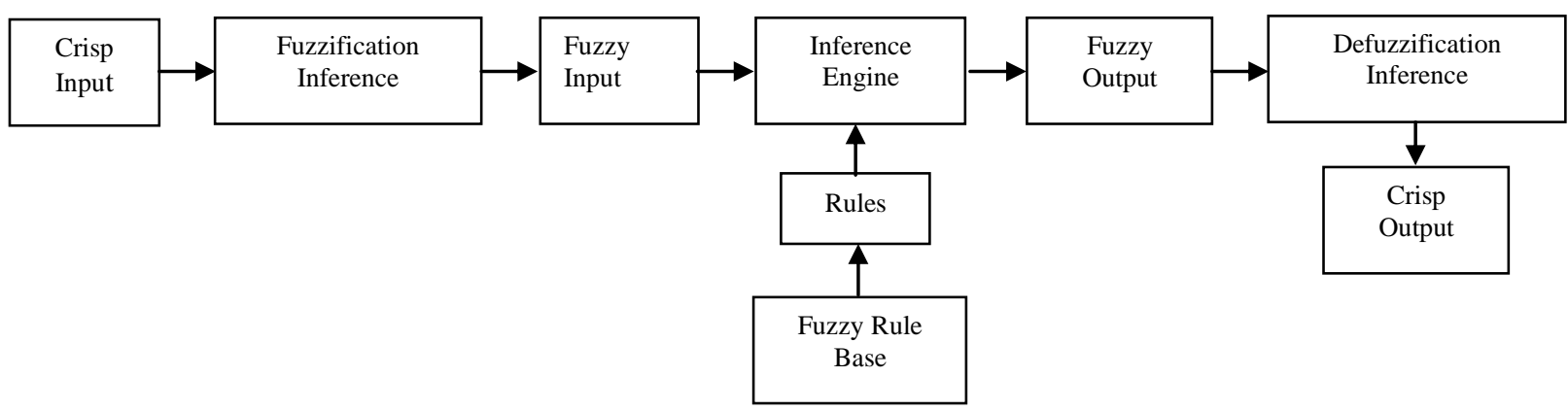

Figure-2: Architecture of Proposed Rule Based Fuzzy Expert System

The main components of proposed rule based fuzzy expert system are: a fuzzification interface, a fuzzy rule-base (knowledge base), an inference engine (decision making logic), and a defuzzification interface [35].

(i) Fuzzification Interface: The input variables are fuzzified by the Fuzzy C-Means clustering algorithm.

(ii) Fuzzy Rule Base (Knowledge Base): Fuzzy if-then rules and fuzzy reasoning are the backbone of fuzzy expert systems, which are the most important modeling tools based on fuzzy set theory. The rule base is characterized in the form of if-then rules in which the antecedents and consequents involve linguistic variables. In this paper, we use very high, high, average, low and very low as linguistic variable. The collection of these rules forms the rule base for the fuzzy logic system. In this proposed rule based fuzzy expert system, we have used the following rules for finding the knowledge base:

1. If student belong to very high then $\left(Y_{1}=a_{1}+b_{1} X\right)$

2. If student belong to high then $\left(Y_{2}=a_{2}+b_{2} X\right)$

3. If student belong to average then $\left(Y_{3}=a_{3}+b_{3} X\right)$

4. If student belong to low then $\left(Y_{4}=a_{4}+b_{4} X\right)$

5. If student belong to very low then $\left(Y_{5}=a_{5}+b_{5} X\right)$

Where $X$ is the students' mark obtained in semester-1 examination. $a_{1}, a_{2}, a_{3}, a_{4}, a_{5}$ and $b_{1}, b_{2}, b_{3}, b_{4}, b_{5}$ are constant determine by the method of regression analysis model.

(iii) Inference Engine (Decision Making Logic): Using suitable inference procedure, the truth value for the antecedent of each rule is computed and applied to the consequent part of each rule. Here, we have used the regression analysis model for decision making. This results in one fuzzy subset to be assigned to each output variable for each rule. Again, by using suitable composition procedure, all the fuzzy subsets to be assigned to each output variable are combined together to form a single fuzzy subset for each output variable.

(iv) Defuzzification Interface: Defuzzification means convert fuzzy output into crisp output. Here, we have used the height defuzzification technique for converting fuzzy output into crisp output (performance value of students). The defuzzification formula (Takagi-Sugeno-Kang Model) is given below:

$$
Y=\frac{\mu_{\text {Very High }}(x) \times Y_{1}+\mu_{\text {High }}(x) \times Y_{2}+\mu_{\text {Average }}(x) \times Y_{3}+\mu_{\text {Low }}(x) \times Y_{4}+\mu_{\text {Very Low }}(x) \times Y_{5}}{\mu_{\text {VeryHigh }}(x)+\mu_{H i g h}(x)+\mu_{\text {Average }}(x)+\mu_{\text {Low }}(x)+\mu_{\text {Very Low }}(x)}
$$

With the help of this equation, we can convert the fuzzy output into crisp output (performance value of a student).

\section{EXPERIMENTAL RESULTS OF PROPOSED RULE BASED FUZZY EXPERT SYSTEM}

In this paper, we have proposed a method called rule based Fuzzy Expert System for academic performance evaluation. We consider here a method by which fuzzy membership function may be created for fuzzy classes of an input data set by using Fuzzy C-Means clustering algorithms. Let us consider, 20 students' marks obtained by Semester-1 and Semester-2 examination. Table-1 shows the scores achieved by 20's B.Tech. $2^{\text {nd }}$ year students in the Department of Computer Science and Engineering, Ashoka Institute of Technology and Management, Saranath, Varanasi-221007, Uttar Pradesh, India, appeared in semester-I and semester-II examination. Here, we 
use the MATLAB software for modeling students' academic performance evaluation.

Table 1. Data Set of Students' Score in Sem.-1 and Sem.-2

\begin{tabular}{|l|c|c|c|c|c|}
\hline S.No. & Sem.-1 & Sem.-2 & S.No. & Sem.-1 & Sem.-2 \\
\hline 1. & 40 & 65 & 11. & 65 & 45 \\
\hline 2. & 20 & 35 & 12. & 89 & 100 \\
\hline 3. & 50 & 65 & 13. & 100 & 100 \\
\hline 4. & 10 & 20 & 14. & 65 & 35 \\
\hline 5. & 45 & 65 & 15. & 48 & 50 \\
\hline 6. & 34 & 60 & 16. & 45 & 55 \\
\hline 7. & 48 & 55 & 17. & 55 & 25 \\
\hline 8. & 56 & 90 & 18. & 84 & 80 \\
\hline 9. & 74 & 70 & 19. & 63 & 65 \\
\hline 10. & 45 & 50 & 20. & 28 & 30 \\
\hline
\end{tabular}

The above data points (Table-4) are first divided into different clusters using Fuzzy C-Means Clustering Techniques. The steps of proposed method are given below:

Step-1 (Fuzzification): Here, we have used Fuzzy C-Means clustering Algorithms for classifying students' scores data set (conversion of crisp score into fuzzy set), given in Table-1. For this purpose, we have used MATLAB software for classifying (Clustering) the students' data score into five classes or Clusters, namely Very High, High, Average, Low, and Very Low for modeling students' academic performance evaluation, shown in Table-2. Figue-2 shows the students dataset partitioned into five classes or clusters. Figue- 4 shows the performance of objective function for students' academic performance evaluation. Table 3 gives the cluster centers of Very High, High, Average, Low and Very Low.

Table-2. The membership functions for fuzzy clustering of Students Academic Performance Evaluation by Fuzzy C-Means Algorithms

\begin{tabular}{|l|c|c|c|c|c|c|c|}
\hline \multirow{2}{*}{ S.No. } & \multirow{2}{*}{ Sem.-1 } & \multirow{2}{*}{ Sem.-2 } & \multicolumn{5}{|c|}{ Fuzzy C-Means Clustering Technique } \\
\cline { 4 - 8 } & & & Very High $(\mathbf{V H})$ & High (H) & Average (A) & Low (L) & Very Low (VL) \\
\hline 1. & 40 & 65 & 0.0138 & 0.0554 & 0.8574 & 0.0412 & 0.0322 \\
\hline 2. & 20 & 35 & 0.0036 & 0.0085 & 0.0290 & 0.0194 & 0.9395 \\
\hline 3. & 50 & 65 & 0.0180 & 0.1135 & 0.7891 & 0.0547 & 0.0247 \\
\hline 4. & 10 & 20 & 0.0115 & 0.0236 & 0.0563 & 0.0517 & 0.8569 \\
\hline 5. & 45 & 65 & 0.0106 & 0.0518 & 0.8862 & 0.0323 & 0.0191 \\
\hline 6. & 34 & 60 & 0.0181 & 0.0610 & 0.7755 & 0.0669 & 0.0784 \\
\hline 7. & 48 & 55 & 0.0054 & 0.0260 & 0.9163 & 0.0379 & 0.0145 \\
\hline 8. & 56 & 90 & 0.1674 & 0.4805 & 0.2206 & 0.0826 & 0.0489 \\
\hline 9. & 74 & 70 & 0.0150 & 0.9490 & 0.0184 & 0.0137 & 0.0039 \\
\hline 10. & 45 & 50 & 0.0120 & 0.0485 & 0.7708 & 0.1161 & 0.0525 \\
\hline 11. & 65 & 45 & 0.0192 & 0.0893 & 0.1196 & 0.7410 & 0.0309 \\
\hline 12. & 89 & 100 & 0.9713 & 0.0176 & 0.0052 & 0.0039 & 0.0019 \\
\hline 13. & 100 & 100 & 0.9518 & 0.0272 & 0.0092 & 0.0079 & 0.0038 \\
\hline 14. & 65 & 35 & 0.0021 & 0.0071 & 0.0107 & 0.9751 & 0.0050 \\
\hline 15. & 48 & 50 & 0.0137 & 0.0595 & 0.7240 & 0.1538 & 0.0491 \\
\hline 16. & 45 & 55 & 0.0029 & 0.0126 & 0.9566 & 0.0186 & 0.0093 \\
\hline 17. & 55 & 25 & 0.0173 & 0.0478 & 0.0975 & 0.7416 & 0.0957 \\
\hline 18. & 84 & 80 & 0.2989 & 0.5613 & 0.0661 & 0.0540 & 0.0197 \\
\hline 19. & 63 & 65 & 0.0364 & 0.6519 & 0.2004 & 0.0875 & 0.0237 \\
\hline 20. & 28 & 30 & 0.0066 & 0.0505 & 0.0543 & 0.0505 & 0.8722 \\
\hline
\end{tabular}

Table 3. The cluster centers of Very High, High, Average, Low and Very Low

\begin{tabular}{|c|c|c|c|}
\hline S.No. & Cluster Center & Sem.-1 & Sem.-2 \\
\hline 1. & Cluster Centre of Very High & 93.2948 & 98.8680 \\
\hline 2. & Cluster Centre of High & 70.5267 & 72.6503 \\
\hline 3. & Cluster Centre of Average & 44.7493 & 58.5596 \\
\hline 4. & Cluster Centre of Low & 61.8312 & 35.7363 \\
\hline 5. & Cluster Centre of Very Low & 19.8020 & 28.9976 \\
\hline
\end{tabular}

The component value of vectors $P$ and $V$ are obtained by soling the fuzzy clustering problem (Academic Performance Evaluation problem), which is basically constrained optimization problems in equation (1). A description of each item of notation as follows:

1. The variable $k$ represents the number of students sit in Semester-1 and Semester-2, who will be allocated into $C$ classes or clusters.

2. The variable $C$ represents the number of classes or clusters, the value of this variable can be determined by the institution policy.

3. The matrix $P=\left(u_{k i}\right)_{n \times c}$ consists of $n$ rows and $c$ columns, of which the element $u_{k i}$ represents the degree of membership (or the suitability level) of the $\mathrm{k}^{\text {th }}$ student.
4. The matrix $v=\left(v_{j i}\right)_{m \times c}$, consists of $m$ rows and $c$ columns, of which the element represents the (weighted) average of students' grade achieved by students, belong to the cluster (or class).

5. In extreme condition, the value of the fundamental $J(P, V)$ equation (10) is 0 , which indicates the obtained clusters are ideal, since they consist of students with the same level of mastery. Principally, the minimum the value of $J(P, V)$ is, then the better the clustering process.

The application of fuzzy C-Means Algorithm (FCM) illustrated by a case described as dataset of students score marks shown in Table-6. Table- 6 gives the value of elements of vector $U_{i}(i=1,2$, $3)$. As an illustration, the values in the $11^{\text {th }}$ row of Table- 6 can be interpreted as: 
Very High $=0.0192$, High $=0.0893$,

Average $=0.1196$, Low $=0.7410$, Very Low $=0.0309$.

$\operatorname{Max}=(0.0192,0.0893,0.1196,0.7410,0.0309=0.7410$

From those five values, $11^{\text {th }}$ student is the most suitable to be in class or cluster (Low), since he/she has the highest degree of membership to this class or cluster compared to the other four. $5^{\text {th }}$ student is the most suitable to be in class or cluster (average), since he/she has the highest degree of membership to this class or cluster compared to the other four. Thus, we conclude that $5^{\text {th }}$ student has improved consistently while $11^{\text {th }}$ student has deteriorated consistently. By the same observations, the following class or cluster was obtained for students partitioning in Semester-1 and Semester-2 examinations:

1. The first class or cluster (Very High) consists of students' numbers 12 , and 13.
2. The second class or cluster (High) consists of students' numbers 8, 9, 18 and 19 .

3. The third class or cluster (Average) consists of students' numbers $1,3,5,6,7,10,15$, and 16 .

4. The fourth class or cluster (Low) consists of students' numbers' 11,14 and 17 .

5. The fifth class or cluster (Very Low) consists of students' numbers 2, 4 and 20.

Thus, two students belong to class or cluster (Very High), four students belong to class or cluster (High), eight students belong to class or cluster (Average), three students belong to class cluster (Low) and three students belong to class or cluster (Very Low).

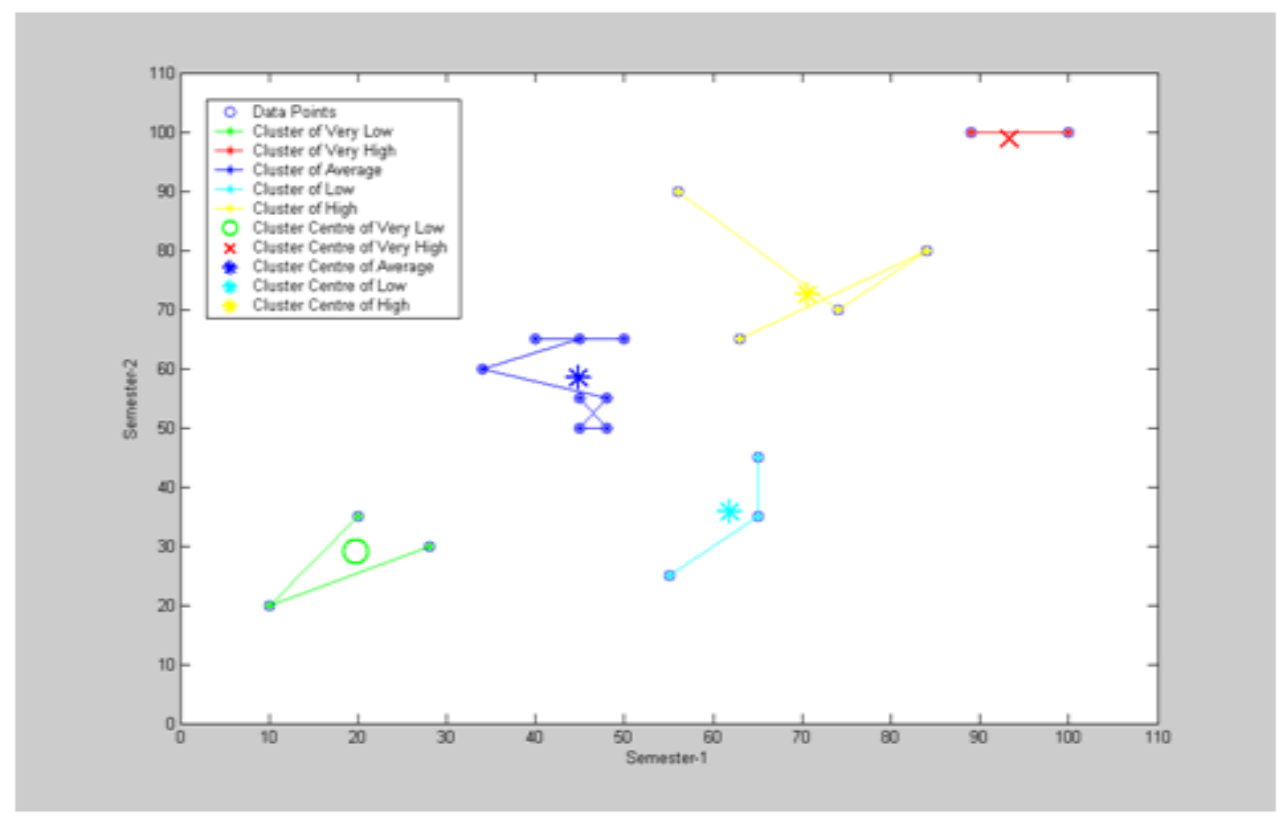

Figure-3: Partition of the students score dataset for academic performance evaluation

\section{Step-2 (Output Estimation):}

Regression problems deal with estimation of an output value based on input values. When used for classification, the input values are values from the database and the output values represents the classes. Regression can be used to solve classification problems. In actually, regression takes a set of data and fits the data to formal. The linear regression formula in two dimensional spaces is given bellow:

$$
y=a+b x
$$

Where $a$ and $b$ are constant. They are determining by the normal equations for best fit of linear relationship of input and output. This model is estimate the actual relationship between input and output. We can use the generated linear regression model to predict an output value given an input value. Here, we use the regression analysis of output estimation of rule based Fuzzy Expert System for modeling academic performance evaluation. In this proposed research work, we have used linear regression model for estimation of output of rule based Fuzzy Expert System. Here we have used the MATAB software for estimating the output of DFES. The output of cluster (Very High), cluster (High), Cluster (Average), cluster (Low) and Cluster (Very Low) are given bellow:

Very High: $Y=100$

High: $Y=89.3843-0.1897 * X$

Average: $Y=71.1063-0.2925 * X$

Low: $Y=-57.5000+1.5000 * X$
Very Low: $Y=16.8443+0.5943 * X$

Where $\mathrm{X}$ is students mark of semester-1.

Step-3 (Rule Generation):

1. If Student belongs to cluster (very high) then student performance is very $\operatorname{high}(Y=100)$.

2. If student is belongs to cluster (high) then student performance is $\operatorname{high}(Y=89.3843-0.1897 * X)$.

3. If student is belongs to cluster (average) then student performance is average $(Y=71.1063-0.2925 * X)$.

4. If student belongs to cluster (low) then student performance low $(Y=-57.5000+1.5000 * X)$.

5. If student belongs to cluster very low then student performance is very low $(Y=16.8443+0.5943 * X)$.

If we take the first student of Table-6, then the output of $Y$ is given by: Very High: $\mathrm{Y}=100$, High: $\mathrm{Y}=89.3843-0.1897 * 40=$ 81.4143, Average: $Y=71.1063-0.2925 * 40=59.4063$, Low: $Y$ $=-57.5000+1.5000 * 40=2.5$, Very Low: $\mathrm{Y}=16.8443-0.5943=$ 40.6163

Step-4 (Defuzzification) Calculation of Academic Performance

The final calculation of student academic performance is determined by the following formula:

$Y=\frac{\mu_{\text {Very High }}(x) \times Y_{1}+\mu_{\text {High }}(x) \times Y_{2}+\mu_{\text {Average }}(x) \times Y_{3}+\mu_{\text {Low }}(x) \times Y_{4}+\mu_{\text {Very Low }}(x) \times Y_{5}}{\mu_{\text {Very }}(x)+\mu_{\text {High }}(x)+\mu_{\text {Average }}(x)+\mu_{\text {Low }}(x)+\mu_{\text {Very Low }}(x)}$ $Y=\frac{100 \times 0.0138+81.4143 \times 0.0554+59.4063 \times 0.8574+4.5 \times 0.0412+40.6163 \times 0.0322}{\mu_{\text {Ver }} \text { High }}=58.257$

Similarly, we can calculate the academic performance of other students' given in Table-4. 


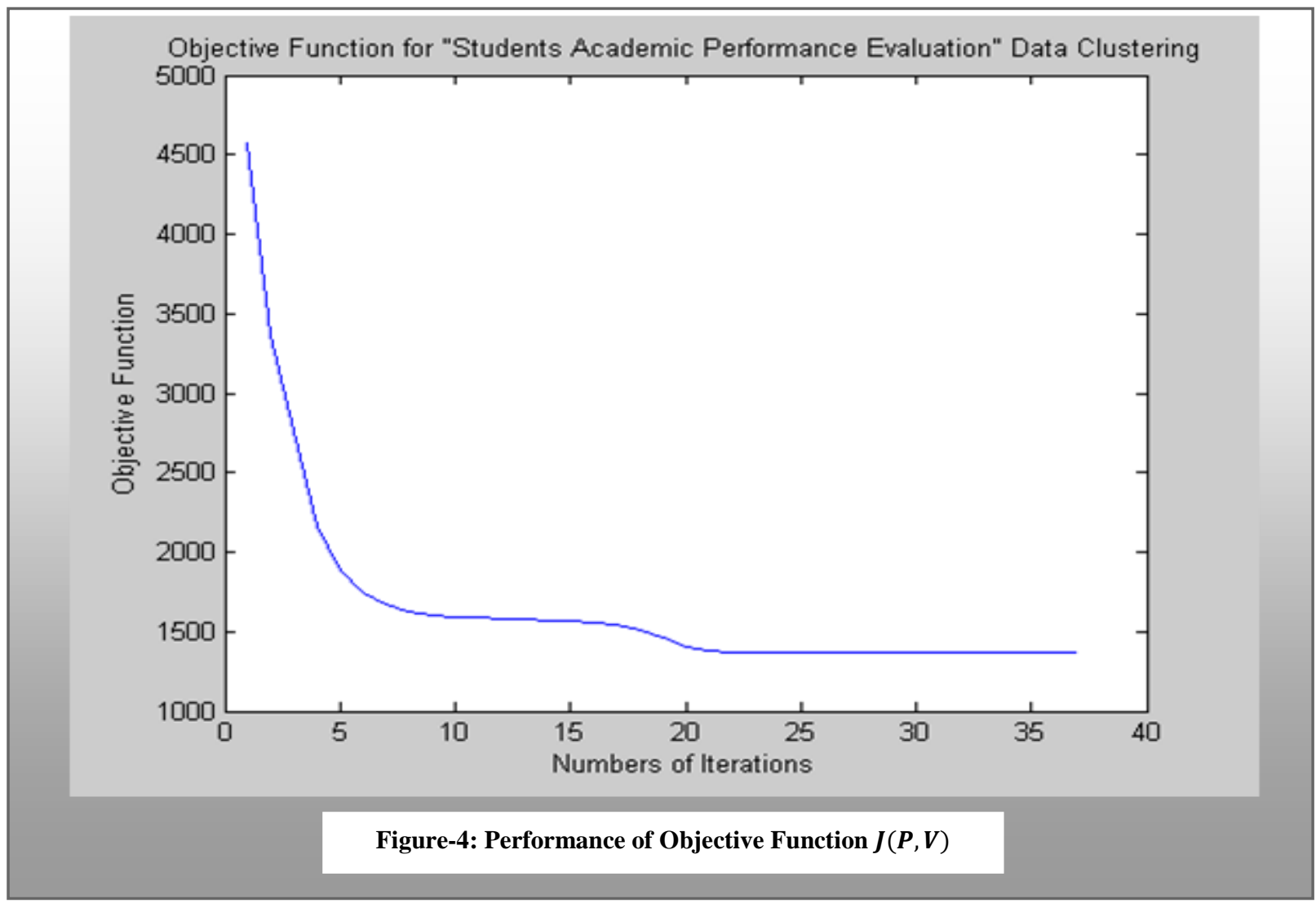

Table-4: The membership functions and Students Academic Performance Calculated by the Rule Based Fuzzy Expert System

\begin{tabular}{|l|c|c|c|c|c|c|c|c|}
\hline S.No. & Sem.-1 & \multirow{2}{*}{ Sem.-2 } & \multicolumn{5}{|c|}{ Student Performance using Rule Based Fuzzy Expert System } \\
\cline { 4 - 9 } & & & $\begin{array}{c}\text { Very High } \\
\text { (VH) }\end{array}$ & $\begin{array}{c}\text { High } \\
(\mathbf{H})\end{array}$ & $\begin{array}{c}\text { Average } \\
(\mathbf{A})\end{array}$ & Low (L) & $\begin{array}{c}\text { Very Low } \\
\text { (VL) }\end{array}$ & $\begin{array}{c}\text { Student } \\
\text { Performance (SP) }\end{array}$ \\
\hline 1. & 40 & 65 & 0.0138 & 0.0554 & 0.8574 & 0.0412 & 0.0322 & 58.257320 \\
\hline 2. & 20 & 35 & 0.0036 & 0.0085 & 0.0290 & 0.0194 & 0.9395 & 29.438568 \\
\hline 3. & 50 & 65 & 0.0180 & 0.1135 & 0.7891 & 0.0547 & 0.0247 & 57.545231 \\
\hline 4. & 10 & 20 & 0.0115 & 0.0236 & 0.0563 & 0.0517 & 0.8569 & 24.382494 \\
\hline 5. & 45 & 65 & 0.0106 & 0.0518 & 0.8862 & 0.0323 & 0.0191 & 57.753239 \\
\hline 6. & 34 & 60 & 0.0181 & 0.0610 & 0.7755 & 0.0669 & 0.0784 & 56.775181 \\
\hline 7. & 48 & 55 & 0.0054 & 0.0260 & 0.9163 & 0.0379 & 0.0145 & 56.118908 \\
\hline 8. & 56 & 90 & 0.1674 & 0.4805 & 0.2206 & 0.0826 & 0.0489 & 71.297348 \\
\hline 9. & 74 & 70 & 0.0150 & 0.9490 & 0.0184 & 0.0137 & 0.0039 & 74.884071 \\
\hline 10. & 45 & 50 & 0.0120 & 0.0485 & 0.7708 & 0.1161 & 0.0525 & 53.238884 \\
\hline 11. & 65 & 45 & 0.0192 & 0.0893 & 0.1196 & 0.7410 & 0.0309 & 46.385464 \\
\hline 12. & 89 & 100 & 0.9713 & 0.0176 & 0.0052 & 0.0039 & 0.0019 & 99.079208 \\
\hline 13. & 100 & 100 & 0.9518 & 0.0272 & 0.0092 & 0.0079 & 0.0038 & 98.510788 \\
\hline 14. & 65 & 35 & 0.0021 & 0.0071 & 0.0107 & 0.9751 & 0.0050 & 40.595856 \\
\hline 15. & 48 & 50 & 0.0137 & 0.0595 & 0.7240 & 0.1538 & 0.0491 & 51.915192 \\
\hline 16. & 45 & 55 & 0.0029 & 0.0126 & 0.9566 & 0.0186 & 0.0093 & 57.329090 \\
\hline 17. & 55 & 25 & 0.0173 & 0.0478 & 0.0975 & 0.7416 & 0.0957 & 34.151695 \\
\hline 18. & 84 & 80 & 0.2989 & 0.5613 & 0.0661 & 0.0540 & 0.0197 & 79.207535 \\
\hline 19. & 63 & 65 & 0.0364 & 0.6519 & 0.2004 & 0.0875 & 0.0237 & 69.206512 \\
\hline 20. & 28 & 30 & 0.0066 & 0.0505 & 0.0543 & 0.0505 & 0.8722 & 35.532959 \\
\hline
\end{tabular}

From above Table- 4 shows that the $11^{\text {th }}$ student is the most suitable to be in class or cluster (Low), since this student has the highest degree of membership to this class or cluster compared to the other four. $5^{\text {th }}$ student is the most suitable to be in class or cluster (average), since this student has the highest degree of membership to this class or cluster compared to the other four. Thus, we conclude that $5^{\text {th }}$ student has improved consistently while $11^{\text {th }}$ student has deteriorated consistently. Therefore, the fuzzy C-Means clustering technique method is more suitable than the classical method for academic performance evaluation. In this model, the numbers of fuzzy rules are very less in comparison to existing classical Fuzzy Expert System. Therefore, the proposed rule based Fuzzy Expert System is more efficient for computational point of view. The proposed rule based Fuzzy Expert System also calculate the total marks of a particular student. For example, $1^{\text {st }}$ student has secured $58.257320,2^{\text {nd }}$ student has secured 29.438568 and $3^{\text {rd }}$ student has secured 57.545231 etc. 


\section{CONCLUSION AND FUTURE WORK}

In this paper, we have proposed rule based Fuzzy Expert system for students' academic performance evaluation based Fuzzy CMeans Clustering Algorithm, Fuzzy Logic and Regression analysis model. The proposed rule based Fuzzy Expert System automatically converted the crisp data into fuzzy set and also calculate the total marks of a student appeared in semsetr- 1 and semester-2 examination.

We have also provided a simple and qualitative methodology to compare the predictive power of clustering algorithm and the Euclidean distance. We demonstrated our technique (Fuzzy CMeans clustering technique) for academic performance evaluation and combined with the deterministic model on a dataset of B.Tech. Students' appeared in semester-1 and semester-2 examination. The Fuzzy C-Means clustering models have improved on some limitation of the existing traditional methods, such as average method and statistical method. We observed that the Fuzzy C-Means algorithm is best model for modeling academic performance in educational domain. Therefore, the fuzzy C-Means clustering algorithm serves as a good benchmark to monitor the progression of students modeling in educational domain. The proposed rule based Fuzzy Expert System is more efficient model in comparison to existing fuzzy expert system for modeling academic performance evaluation.

It also enhances the decision making by academic planners semester by semester by improving on the future academic results in the subsequence academic session. It worth of future research to use combine technique of fuzzy C-Means, Artificial Neural Networks called Neuro-Dynamic Fuzzy Expert system to evaluate student and teacher academic performance and also develop adaptive learning system and Intelligent Tutoring System for Internet based education like Distance Education. This system is implemented by using the Fuzzy Logic Toolbox $^{\text {TM }} 2.2 .7$ by MathWorks.

\section{ACKNOWLEDGEMENTS}

I would like to express my deep sense of gratitude and respect to my supervisor Prof. Pervez Ahmed, for their excellent guidance and suggestions. They have been to source of inspiration for me. I would like to render heartiest thanks to various friends for their priceless help and support. Last but not the least we thank our parents and wife and the almighty whose blessings are always there with us.

\section{REFERENCES}

[1] Mankad, K., Sajja, P. S., \& Akerkar, R. (2011). Evolving Rules Using Genetic Fuzzy Approach: An educational case study. International Journal on Soft Computing. 2(1), 3546.

[2] Biswas, R. (1995). An Application of fuzzy sets in Students' Evaluation. Fuzzy sets and System, ELSEVIER, 187-194.

[3] Zadeh, L.A. (1965). Fuzzy sets. Information and Control, $8,338-354$

[4] Wang, H.Y., \& Chen, S.M. (2007). Artificial Intelligence Approach to Evaluate Students' Answerscripts Based on the Similarity Measure Between Vague Sets. Educational Technology and Society, 10(4), 224-241.

[5] Gau, W.L. \& Buehrer, D.J. (1993). Vague Sets. IEEE Transactions on System. Man and Cybernatics, 23(2), 610614.

[6] Bai, S.M., \& Chen, S.M. (2008). Evaluating Students' Learning Achievement Using Fuzzy membership functions and Fuzzy rules. Expert System with Applications, ELSEVIER, 34, 399-410.

[7] Law, C.K. (1996). Using Fuzzy Numbers in Educational Grading system. Fuzzy sets and System 83, 311-323.

[8] Chen, S.M., \& Lee, C.H. (1999). New Methods for Students' Evaluation Using Fuzzy Sets. Fuzzy Sets and System, 104, 209-218.

[9] Wang, H.Y, \& Chen, S.M. (2006). New Methods for Evaluating Students Answerscripts Using Fuzzy Numbers Associated with Degrees of Confidence. 2006 IEEE International Conference on Fuzzy Systems, 1004-1009.

[10] Stathacopoulou, R., Magoulas, G.D., Grigoriadou, M. \& Samarakou (2005). Neuro-Fuzzy Knowledge Processing in Intelligent Learning Environments for Improved Student Diagnosis. Information Science, ELSEVIER, 170(2-4), 273-307.

[11] Guh, Y.Y., Yang, M.S., Po, R.W., \& Lee, E.S. (2008) Establishing Performance Evaluation Structures by Fuzzy Relation Based Cluster Analysis. Computers and Mathematics Applications, 56, 572-582.

[12] Gokmen, E., Akinci, T.C., Tektas, M., Onat, N., Kocyigit, G., \& Tektas, N. (2010). Evaluation of Student Performance in Laboratory Applications Using Fuzzy Logic. Procedia Social and Behavioral Science, 2, 902-909.

[13] Hameed, I.A. (2011). Using Gaussian Membership Functions for Improving the Reliability and Robustness of Students' Evaluation System. International Journal of Expert System with Applications, 38 (6), 7135-7142.

[14] Baylari, A., \& Montazer, G. A. (2009). Design a Personalized E-learning System Based on Item Response Theory and Artificial Neural Network Approach. Expert System with Applications, 36, 8013-8021.

[15] Posey, C.L., \& Hawkes, L.W. (1996). Neural Networks Applied in the Student Model. Intelligent Systems, 88, 275-298.

[16] Stathacopoulou, R., Grigoriadou, M., Samarakou, M., \& Mitoropoulou , D. (2007). Monitoring Students' Action and Using Teachers' Expertise in Implementing and Evaluating the Neural Network-based Fuzzy Diagnostic Model. Expert Systems with Applications, 32, 955-975.

[17] Bhatt, R., \& Bhatt, D. (2011). Fuzzy Logic Based Student Performance Evaluation Model for Practical Components of Engineering Institutions Subjects. International Journal of Technology and Engineering Education,8(1), 1-7.

[18] Gupta, C.R., \& Dhawan, A.K. (2012). Diagnosis, Modeling and Prognosis of Learning System Using Fuzzy Logic and Intelligent Decision Vectors. International Journal of Computer Applications, 37(6), (975-987).

[19] Ma, J., \& Zhou, D. (2000). Fuzzy Set Approach to the Assessment of student Centered Learning. IEEE Transaction on Education, 43(2), 112-120.

[20] Krzysztof, J., Cios, Pedrycz, W., Swiniarski, R. W., Lukasz, A., \& Kurgan (2007). Data Mining: A Knowledge Discovery Approach. Springer, 263-265.

[21] Gagula-Palalic, S., \& Can, M. (2008). Fuzzy Clustering Models and Algorithms for Pattern Recognition. Master Thesis, 13-17. 
[22] Yen, J., \& Langari, R. (1999). Fuzzy Logic: Intelligence, Control and Information. Center for Fuzzy logic. Robotics and Intelligent Systems. Texas A \& M University, 375401.

[23] S. S. Sansgiry, M. Bhosle and K. Sail (2006). Factors that Affect Academic Performance among Pharmacy Students. American Journal of Pharmaceutical Education, (231-243).

[24] Oyelade, O.J., Oladipupo, O.O., \& I.C. Obagbua (2010). Application of K-Means Clustering Algorithm for prediction of students' Academic Performance. International Journal of Computer Science and Information Security. 7(1), 292-295.

[25] Zukhri, Z., \& Omar, K. (2008). Solving New Student Allocation Problem with Genetic Algorithm: A Hard Problem for Partition Based Approach. International Journal of Soft Computing Applications. Euro Journal Publishing Inc., 6-15.

[26] Pavani, S., Gangadhar, P.V.S.S., \& Gulhare, K. K. (2012). Evaluation of Teacher's Performance Evaluation Using Fuzzy Logic Techniques. International Journal of Computer Trends and Technology. 3(2), 200-205.

[27] Sreenivasarao, V., \& Yohannes, G. (2012). Improving Academic Performance of Students of Defense University Based on data Warehousing and Data Mining. Global Journal of Computer Science and Technology. 12(2), 201209.

[28] Afoayan, O., \& El-Shamir Absalom, E. (2010). Design and implementation of Student's Information System for Tertiary Institutions Using Neural Network Techniques. International Journal of Green Computing. 1(1), (1-15)

[29] Chaudhari O.K., Khot, P.G., \& Deshmukh, K.C. (2012). Soft Computing Model for Academic Performance of
Teachers Using Fuzzy Logic. British Journal of Applied Science and Technology. 2(2), 213-226.

[30] Neogi, A., Mondal, A. C., \& Mandal, S. (2011). A Cascaded Fuzzy Inference System for University NonTeaching Staff Performance Appraisal. Journal of Information Processing Systems, 7(4), 595-612.

[31] Yadav, R. S., \& Singh, V. P. (2011). Modeling Academic Performance Evaluation Using Soft Computing Techniques: A Fuzzy Logic Approach. International Journal on Computer Science and Engineering, 3(2), 676686.

[32] Daud, W. S. W., Aziz, K. A. A., \& Sakib, E. (2011). An Evaluation of Students' Performance in Oral Presentation Using Fuzzy Approach. Empowering Science, Technology and Innovation towards a Better Tomorrow. UMTAS 2011, (MO36), 157-162.

[33] Upadhyay, M. S. (2012). Fuzzy Logic Based of Performance of Students in College. Journal of Computer Applications (JCA), 5(1), 6-9.

[34] White, H. (1989). Learning in Artificial Neural Networks: A Statistical Perspective. Neural Computation, 1, 425-464.

[35] Giarratano, J.C. \& Riley, G. (2005). Expert System: Principles and Programming. Fourth ed., PWS Publishing Com. Boston, MA, USA

[36] Schneider, M., Langholz, G., Kandel, A., \& Chew, G. (1996). Fuzzy Expert System Tools, Jhon Willy and Sons, USA.

[37] www.brighthub.com, 2010. 